\title{
Knockdown of RhoA expression alters ovarian cancer biological behavior in vitro and in nude mice
}

\author{
XIAOXIA WANG ${ }^{1}$, WENYAN JIANG ${ }^{2}$, JIALI KANG ${ }^{2}$, QICAI LIU $^{3}$ and MIAOLING NIE ${ }^{2}$ \\ ${ }^{1}$ Department of Obstetrics and Gynecology, The First Affiliated Hospital of Jinan University, Guangzhou, \\ Guangdong 510630; ${ }^{2}$ Department of Obstetrics and Gynecology, Guangzhou First People's Hospital Affiliated \\ to Guangzhou Medical University, Guangzhou, Guangdong 510180; ${ }^{3}$ Experimental Medical Research Center, \\ Guangzhou Medical University, Guangzhou, Guangdong 510182, P.R. China
}

Received February 4, 2015; Accepted April 28, 2015

DOI: $10.3892 /$ or.2015.4009

\begin{abstract}
RhoA regulates cell proliferation, migration, angiogenesis and gene expression. Altered RhoA activity contributes to cancer progression. The present study investigated the effects of RhoA knockdown on the regulation of ovarian cancer biological behavior in vitro and in nude mice. The expression of RhoA was knocked down using a lentivirus carrying RhoA short hairpin RNA (shRNA) in ovarian cancer cells and was confirmed by reverse transcription-quantitative polymerase chain reaction (RT-qPCR) and western blot analysis. The altered ovarian cancer biological behaviors were assayed by cell viability, terminal deoxynucleotidyltransferase-mediated dUTP nick end-labeling (TUNEL), migration, invasion, and nude mice tumorigenicity assays, while the altered gene expression was detected by RT-qPCR and western blot analysis. The results showed that lentivirus-carrying RhoA shRNA significantly suppressed RhoA expression in ovarian cancer cells, which suppressed tumor cell viability, migration, invasion and adhesion in vitro. RhoA silencing also inhibited the tumorigenicity of ovarian cancer cells in nude mice, which was characterized by the suppression of tumor xenograft formation and growth and induction of tumor cell apoptosis. The results of the present study demonstrated that knockdown of RhoA expression had a significant antitumor effect on ovarian cancer cells in vitro and in nude mice, suggesting that RhoA may be a target for the development of a novel therapeutic strategy in the control of ovarian cancer.
\end{abstract}

Correspondence to: Professor Jiali Kang, Department of Obstetrics and Gynecology, Guangzhou First People's Hospital Affiliated to Guangzhou Medical University, 1 Panfu Road, Guangzhou, Guangdong 510180, P.R. China

E-mail: kangjiali1961@21cn.com

Key words: ovarian cancer, RhoA, gene knockdown, cell viability, migration and invasion, apoptosis, tumor cell xenograft model

\section{Introduction}

Epithelial ovarian carcinoma is a significant health problem in women, accounting for $\sim 160,000$ cancer-related mortalities in 2010 worldwide (1). Ovarian cancer is especially more common in industrialized nations, with the exception of Japan (1), and the risk factors include hormone therapy after menopause, gene mutations (BRCA1 or BRCA2), tobacco smoking and obesity. The risk also increases with age but decreases with the number of pregnancies (2-4). Most ovarian types of cancer are diagnosed at the advanced stages of disease and lead to a poor prognosis (5). Despite surgical resection coupled with systemic chemotherapy and radiotherapy, the 5-year survival rate of ovarian cancer remains $\sim 30 \%$, and most patients succumb to this disease due to tumor recurrence and metastasis (6). Thus, our research efforts on ovarian cancer, as with most other cancer types, should focus on controlling cancer metastasis, which includes several essential steps, i.e., tumor cell proliferation, migration, invasion, adhesion, and formation of metastasis in adjacent or distant organs or tissues $(7,8)$. At the gene level, RhoA, a member of the Ras superfamily, acts as a molecular switch to promote cell mobility and, therefore, to participate in tumor progression. Specifically, RhoA activation can lead to a diverse set of biological responses, including cell motility, proliferation, apoptosis inhibition, cell cycle progression, invasion, and metastasis of tumor cells (9-12). Accumulating evidence has shown that RhoA activity is upregulated in most human carcinogenesis $(13,14)$ and tumor progression $(15,16)$. Thus, RhoA may be a novel molecular target for controlling cancer metastasis.

It was previously demonstrated that the negative regulation of RhoA translation and signaling affected cell morphogenesis (17), and the knockdown of RhoA expression using adenovirus-mediated RNA interference inhibited the proliferation and invasive ability of lung, gastric and colorectal cancer cells in vitro $(18,19)$. Thus, in the present study, we utilized a lentivirus-carrying RhoA short hairpin RNA (shRNA) to knock down RhoA expression in a highly invasive epithelial ovarian cancer cell line and then assessed the altered biological behaviors in vitro and in nude mice. 


\section{Materials and methods}

Cell lines and culture. The human HO8910 epithelial ovarian cancer cell line and human 293FT embryonic kidney cell line were obtained from the Zhongshan University Laboratory Animal Center (Guangzhou, China). HO8910 cells were grown in Roswell Park Memorial Institute (RPMI)-1640 medium (Invitrogen-Life Technologies, Carlsbad, CA, USA) supplemented with $10 \%$ fetal bovine serum (FBS; Hyclone, Logan, UT, USA), while 293FT cells were cultured in Dulbecco's modified Eagle's medium supplemented with $4.5 \mathrm{~g} / 1$ glucose (PAA Laboratories, Pasching, Austria), $10 \%$ heat-inactivated FBS (Hyclone), $100 \mathrm{U} / \mathrm{ml}$ penicillin and $100 \mu \mathrm{g} / \mathrm{ml}$ streptomycin (both from Invitrogen-Life Technologies) in a humidified incubator containing $5 \% \mathrm{CO}_{2}$ at $37^{\circ} \mathrm{C}$.

Establishment of stable RhoA-knockdown (RhoA-KD) tumor cells using a lentivirus-carrying RhoA shRNA. A lentiviral expression vector carrying RhoA shRNA was obtained from GeneCopoeia (Rockville, MD, USA), and the target sequence of RhoA cDNA was 5'-GAAGGCAGAGATATGGCAA-3'. A negative control vector was provided by GeneCopoeia, designated as Lenti-RhoA-NC. Lenti-RhoA-sh and Lenti-RhoA-NC vectors contained enhanced green fluorescent protein (eGFP) cDNA. The recombinant and control lentivirus were then produced via the co-transfection of 293FT cells with the Lenti-Pac $^{\mathrm{TM}}$ HIV Expression Packaging kit (GeneCopoeia), and the virus-containing supernatant was harvested at 48 and $72 \mathrm{~h}$ post infection. To stably establish the RhoA-knockdown HO8910 subline, cells were grown and infected with lentivirus at a multiplicity of infection of 50, and the infection efficiency was directly measured by the detection of eGFP expression in cells by fluorescence microscopy. The cells were grown in medium containing $1.5 \mu \mathrm{g} / \mathrm{ml}$ puromycin (Invitrogen) for 20 days. After reverse transcription-quantitative polymerase chain reaction (RT-qPCR) and western blot analysis confirmation of RhoA knockdown, RhoA shRNA-lentivirus-infected cells were named as RhoA-KD cells, whereas the control Lenti-RhoA-NC-infected cells were designated as mock cells.

$R T-q P C R$. Total cell RNA was isolated from ovarian cancer cells using TRIzol reagent (Invitrogen-Life Technologies) and reverse transcribed into cDNA using the PrimeScript RT-PCR kit (Takara Bio Inc., Shiga, Japan), according to the manufacturer's instructions. The primers for the human RhoA gene were: forward, 5'-TTCCATCGACAGCCCTGATAGTTTA-3' and reverse, 5'-CACGTTGGGACAGAAATGCTTG-3'; while GAPDH was used as the internal control with the primers: forward, 5'-GCACCGTCAAGGCTGAGAAC-3' and reverse, 5'-TGGTGAAGACGCCAGTGGA-3'. PCR amplification and real-time fluorescence signaling monitoring was conducted using a Stratagene fluorescence quantitative PCR instrument according to a previous study (20).

Protein extraction and western blot analysis. Cell lysis was performed using RIPA lysis buffer from Santa Cruz Biotechnology, Inc. (Santa Cruz, CA, USA), and the protein concentration was measured using the Bradford method with the BCA protein assay kit (Pierce Biotechnology, Rockford, IL, USA). For western blot analysis, equal amounts of protein extracts $(30 \mu \mathrm{g})$ were separated by $12 \%$ sodium dodecyl sulfate-polyacrylamide gel electrophoresis and then transferred onto $0.45-\mu \mathrm{m}$ polyvinylidene difluoride membranes (Millipore, Billerica, MA, USA). The membranes were blocked in 5\% non-fat dry milk solution in Tris-based saline Tween-20 (TBST) for $1 \mathrm{~h}$ and then incubated at $4^{\circ} \mathrm{C}$ overnight with a polyclonal rabbit anti-RhoA antibody at a dilution of 1:100 (no. sc-179; Santa Cruz Biotechnology, Inc.) or a rabbit anti- $\beta$-actin antibody at a dilution of 1:1,000 (no. AP0060; Bioworld Technology, Louis Park, MN, USA) in TBST containing $5 \%$ non-fat dry milk, followed by incubation with a secondary horseradish peroxidase-conjugated anti-rabbit antibody (no. BS13278; Bioworld Technology) at a dilution of 1:5,000 in TBST containing 5\% non-fat dry milk for $1 \mathrm{~h}$ at room temperature. The target protein bands were visualized using an enhanced chemiluminescence detection system (Pierce Biotechnology) and quantified using Image J 1.44p software (National Institutes of Health, Bethesda, MD, USA).

Cell viability assay. The altered cell viability after RhoA expression was knocked down in ovarian cancer cells was assayed by the cell viability 3-(4,5-dimethylthiazol2-yl)-2,5-diphenyltetrazolium bromide (MTT) assay as previously described (21). Stable RhoA-knockdown or control shRNA-infected cells were seeded in 96-well plates at a density of $5 \times 10^{3}$ cells/well and grown for up to 5 days. At the end of each experiment, $1 \mu \mathrm{l}$ of MTT solution (Sigma-Aldrich, St. Louis, MO, USA) was added into each well and the plates were incubated for an additional $4-\mathrm{h}$ at $37^{\circ} \mathrm{C}$. Then, $100 \mu \mathrm{l}$ of dimethyl sulfoxide (DMSO) was added into each well to dissolve formazan by agitation for $10 \mathrm{~min}$. The absorbance values (A) of cells were measured at a wavelength of $492 \mathrm{~nm}$ using a microplate reader. The experiments were performed in 5 wells and were repeated at least three times. The data were summarized as a percentage of the control using the formula: $\left(\mathrm{OD}_{\mathrm{RhoA}} / \mathrm{OD}_{\text {control }}\right) \times 100$, where $\mathrm{OD}_{\mathrm{RhoA}}$ and $\mathrm{OD}_{\text {control }}$ were the optical densities in RhoA-knockdown and negative control cultures, respectively.

Cell migration and invasion assay. Tumor cell migration and invasion ability were assayed using a 24-well Transwell chamber system with an $8-\mu \mathrm{m}$ pore size polycarbonate membrane (Corning Life Sciences, Corning, NY, USA), as previously described $(22,23)$. The difference between the tumor cell migration and invasion ability experiments was that the polycarbonate membrane was either pre-coated or not with Matrigel (30 $\mu \mathrm{l}$, diluted at 1:3 with cell culture medium; BD Biosciences, San Jose, CA, USA). For the two assays, the cells were starved for $24 \mathrm{~h}$ and collected. Cells $\left(5 \times 10^{4}\right)$ in $200 \mu \mathrm{l}$ of growth medium containing $0.5 \%$ bovine serum albumin (BSA; Sigma-Aldrich) were then placed in the upper chamber. The lower chamber was filled with $600 \mu \mathrm{l}$ of growth medium containing $20 \% \mathrm{FBS}$, and the cells were cultured at $37^{\circ} \mathrm{C}$ for $48 \mathrm{~h}$. At the end of the experiments, the cells on the upper surface of the filter were removed using a cotton swab, while the cells that migrated or invaded through the filter or Matrigel-coated filter on the lower surface were fixed with $4 \%$ neutral-buffered formalin and stained in $0.01 \%$ crystal violet solution. The cell numbers were counted in 5 fields (up, down, middle, left and right; magnification x200) for each chamber, 
and the results were presented as the mean value \pm standard deviation (SD). The experiment was performed in triplicate and repeated three times.

Cell adhesion assay. The cell adhesion ability was assayed using a 96-well plate that was pre-coated with $30 \mu$ l of Matrigel (diluted at 1:3 with growth medium). Particularly, cells were seeded on the plate and incubated in growth medium containing $0.1 \%$ BSA overnight. The following day, the cells were washed three times with serum-free medium, resuspended at a concentration of $1 \times 10^{6} / \mathrm{ml}$ in serum-free medium, seeded into each well (100 $\mu \mathrm{l} /$ well), and then incubated at $37^{\circ} \mathrm{C}$ for $1 \mathrm{~h}$. Subsequently, the detached cells were washed away with phosphate-buffered saline (PBS), $10 \mu \mathrm{l}$ of MTT solution was added to each well containing attached cells, and the plates were incubated at $37^{\circ} \mathrm{C}$ for $4 \mathrm{~h}$. DMSO $(100 \mu \mathrm{l})$ was added into each well to dissolve formazan, and the absorbance value was measured at a wavelength of $492 \mathrm{~nm}$ using a microplate reader. The data were summarized and presented as the mean value $\pm \mathrm{SD}$, and the tumor cell adhesion rate was calculated using the following formula: relative adhesion rate $(\%)=\left(\mathrm{A}_{492}\right.$ of experimental group $/ \mathrm{A}_{492}$ of the control group) $\mathrm{x} 100 \%$. Each experiment was performed in triplicate and repeated three times.

Tumor cell intraperitoneal tumorigenicity assay in nude mice. Athymic nude female BALBC/c mice (4-6 weeks old, $14.96 \pm 0.96 \mathrm{~g}$, animal protocol number: 0113061) were obtained from the Guangdong Medical Laboratory Animal Center (Guangzhou, China) [registration no.SCXK(YUE)2008-0002], maintained in specific pathogen-free, temperature-controlled isolation conditions, and fed with sterilized food and autoclaved water. Animal breeding, care, and experimental procedures were approved by the Ethics and Human Committee of Guangzhou Medical University and carried out strictly in accordance with the related regulations on the use of experimental animals.

To evaluate tumor cell peritoneal metastasis ability, 21 athymic nude female BALBC/c mice were randomly assigned to three groups ( $n=7$ per group): i) RhoA-KD xenograft, ii) mock xenograft, and iii) parental xenograft groups. Cells in the logarithmic growth phase were collected and washed twice with PBS. Cells (5.0x10\%/mouse) in $200 \mu 1$ of serum-free medium with a survival rate of $>95 \%$ (assessed using trypan blue staining) were injected into the peritoneal cavity of each mouse. The mice were monitored every 2 days and sacrificed 28 days after tumor cell inoculation. The abdominal circumference and ascetic volume were measured. The number of tumor lesions and disseminated tumors were counted, and the tumor nodules were excised for histopathology, RT-qPCR, western blot analysis, immunohistochemical staining, and terminal deoxynucleotidyltransferase-mediated dUTP nick end-labeling (TUNEL) assays. The liver, spleen, lung, and renal tissues were taken and used for the histopathological analysis of tumor metastasis.

Histopathology and immunohistochemistry. Mouse tumor tissues were fixed in $4 \%$ paraformaldehyde for $24 \mathrm{~h}$ and embedded in paraffin. Tissue sections $(4 \mu \mathrm{m})$ were prepared and stained with hematoxylin and eosin. The consecutive sections were used for immunohistochemical staining.
Specifically, the sections were dewaxed in toluene, rehydrated, permeabilized in citrate buffer ( $\mathrm{pH} \mathrm{6.0)}$, and quenched by $3 \%$ $\mathrm{H}_{2} \mathrm{O}_{2}$ for 15 min to inhibit any endogenous peroxidase activities. The sections were then incubated overnight at $4{ }^{\circ} \mathrm{C}$ with a polyclonal rabbit anti-RhoA antibody (diluted at 1:100). The following day, the sections were washed with PBS three times and then incubated with a biotinylated goat anti-rabbit IgG (Dako, Glostrup, Denmark), followed by peroxidase-conjugated streptavidin. The colorimetric reaction was performed using diaminobenzidine, and the sections were counterstained with hematoxylin. Immunostained sections were evaluated by two independent observers who were blinded to the tissue identity. The staining was scored as positive vs. negative when $\geq 10 \%$ tumor cells were positively stained with the polyclonal rabbit anti-RhoA antibody.

TUNEL assay. Immunohistochemical detection and quantification of apoptosis of mouse xenograft tumor tissues were performed using an In Situ Cell Death Detection kit conjugated with horseradish peroxidase (Roche Applied Science, Indianapolis, IN, USA), according to the manufacturer's instructions. The level of apoptosis was evaluated by counting the TUNEL-positive cells (brown-stained). The apoptotic index was determined as the number of TUNEL-positive cells/total number of cells in five randomly selected high-power fields (magnification, x400).

Statistical analysis. Data were presented as mean \pm SD. Statistical analysis was performed using SPSS 13.0 statistical software (SPSS, Inc., Chicago, IL, USA). Differences among different groups were assessed using one-way analysis of variance, and differences between two groups were assessed using the Student-Newman-Keuls (SNK) test. $\mathrm{P}<0.05$ was considered statistically significant.

\section{Results}

Stable knockdown of RhoA expression in ovarian cancer cells using a lentivirus-carrying RhoA shRNA. In the present study, we assessed the role of RhoA knockdown in ovarian cancer cells. HO8910 cells infected with lentivirus for $48 \mathrm{~h}$ showed an infection efficiency of $60 \%$ by fluorescence microscopy (Fig. 1A). After the cells were cultured in puromycin $(1.5 \mu \mathrm{g} / \mathrm{ml})$-containing growth medium for 20 days, stable RhoA-KD and mock ovarian cancer cell populations were successfully generated (Fig. 1A). Although these cell populations were cultured and passaged $>10$ times, the eGFPpositive expression in the cells remained $>98 \%$. The RT-qPCR and western blot analysis confirmed that the RhoA-KD cells had a significantly decreased expression of RhoA mRNA and protein (Fig. 1B and $\mathrm{C}, \mathrm{P}<0.05$ ). Moreover, there was no difference in the RhoA expression between parental HO8910 cells and the mock cells (Fig. 1B and C, P>0.05).

Effects of RhoA knockdown on the regulation of HO8910 cell biological behaviors in vitro. After the stable RhoA-knockdown ovarian cancer cell population was obtained, we first assayed the effects of RhoA silencing on the regulation of tumor cell viability. As shown in Fig. 2A, although there was not a statistical difference in the cell viability among the parental, 


\section{A Fluorescence microscope}
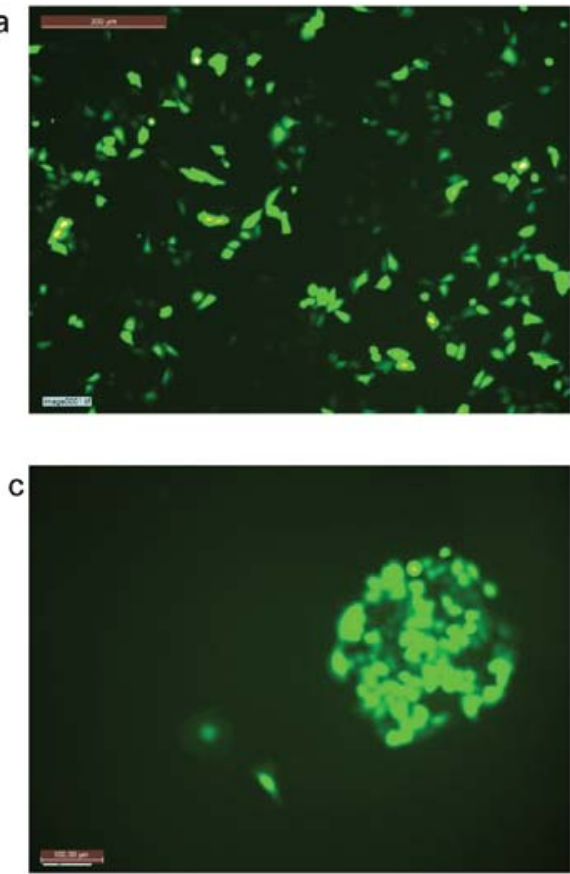

B

RT-qPCR

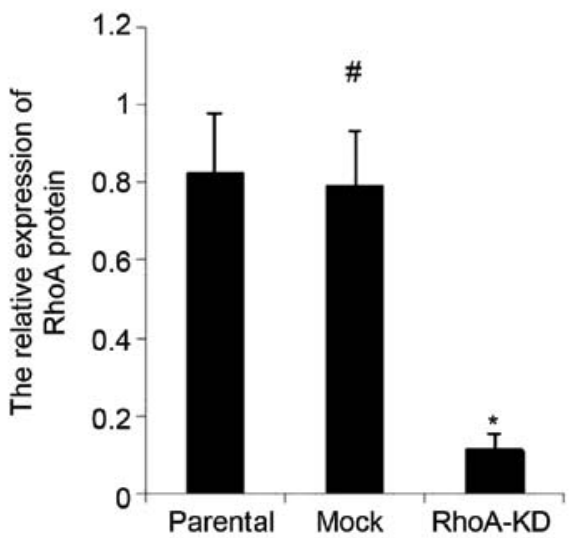

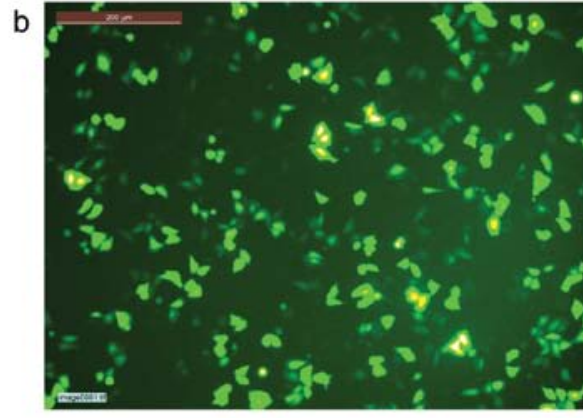

d



C Western blot

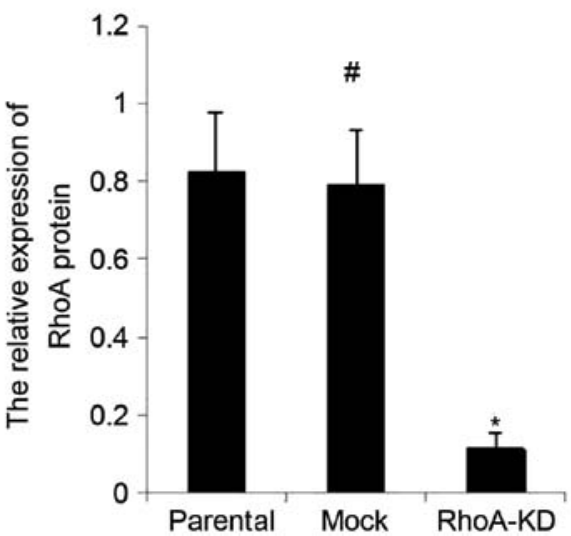

Figure 1. Stable knockdown of RhoA expression in ovarian cancer HO8910 cells using a lentivirus-carrying RhoA shRNA. (A) Fluorescence microscopy. (a) HO8910 cells were infected with Lenti-RhoA-sh for $48 \mathrm{~h}$ and reviewed under a fluorescence microscope (magnification, x100); (b) HO8910 cells were infected with Lenti-RhoA-NC for $48 \mathrm{~h}$ and reviewed under a fluorescence microscope (magnification, $\mathrm{x} 100$ ); (c) Stable RhoA-KD HO8910 cells were grown in selection medium for 20 days and reviewed under a fluorescence microscope (magnification, x200); (d) Stable negative control mock HO8910 cells were grown in selection medium for 20 days and reviewed under a fluorescence microscope (magnification, x200). (B) RT-qPCR. Stable RhoA-KD, mock and parental HO8910 cells were grown and subjected to RT-qPCR analysis of RhoA expression. (C) Western blot analysis. Stable RhoA-KD, mock and parental HO8910 cells were grown and subjected to western blot analysis of RhoA expression. Data are presented as the mean $\pm \mathrm{SD} .{ }^{*} \mathrm{P}<0.05$ vs. parental and mock cells; ${ }^{*} \mathrm{P}>0.05$ vs. parental cells. shRNA, short hairpin RNA; RhoA-KD, RhoA-knockdown; RT-qPCR, reverse transcription-quantitative polymerase chain reaction.

mock, and RhoA-KD cell cultures on day $1(\mathrm{P}>0.05)$, the cell viability of the RhoA-KD cultures was significantly attenuated between day 2 and $5(\mathrm{P}<0.05)$. By contrast, there was no difference in cell viability between the parental HO8910 and mock tumor cells $(\mathrm{P}>0.05)$. Moreover, the tumor cell migration and invasion assays showed that the knockdown of RhoA expression reduced the tumor cell migration and invasion abilities compared with the mock and parental HO8910 cells $(\mathrm{P}<0.05)$, whereas the parental HO8910 and mock cells showed similar migration and invasion abilities (Fig. $2 \mathrm{~B}$ and $\mathrm{C}, \mathrm{P}>0.05$ ).

Cancer cell adhesion to the peritoneum is a crucial process and the initial step during ovarian cancer peritoneal metastasis.
Therefore, we assessed the effects of the knockdown of RhoA expression on the regulation of tumor cell adhesion. As shown in Fig. 3, the tumor cell adhesion was markedly decreased after RhoA knockdown compared to that of the parental HO8910 and mock cells $(\mathrm{P}<0.05)$. However, the mock and parental HO8910 cells showed similar cell adhesion activities $(\mathrm{P}>0.05)$.

Effects of RhoA knockdown on the regulation of HO8910 cell biological behaviors in nude mice. We assessed the in vivo effect of RhoA silencing in nude mice. We found that 10 days after tumor cell inoculation, the mice with parental or mock cell injections exhibited gradually decreased activity and appeared 
A Cell viability MTT assay



B Matrigel invasion assay



C Transwell migration assay



Figure 2. Effects of RhoA knockdown on the regulation of tumor cell viability, migration, and invasion ability. (A) Cell viability MTT assay. Stable RhoA-KD, mock and parental HO8910 cells were grown and subjected to the MTT assay. The data showed that the A492 values of RhoA-KD cells were significantly decreased starting at day $2(\mathrm{P}<0.05)$. Data are presented as the mean $\pm \mathrm{SD}$ of three independent experiments. ${ }^{*} \mathrm{P}<0.05$ vs. parental and mock cells; ${ }^{*} \mathrm{P}>0.05$. However, there was no significant difference in cell viability among the three groups on the first day. (B) Matrigel invasion and (C) Transwell migration assays. Stable RhoA-KD, mock and parental HO8910 cells were grown and then subjected to the tumor cell migration or invasion assay. The results show that there was a statistically significant decrease in the number of invading and migrating cells in the RhoA-KD cells compared to those of the parental and mock cells. Data are presented as the mean $\pm \mathrm{SD}$ of three independent experiments. " $\mathrm{P}<0.05$ vs. parental and mock cells. ${ }^{"} \mathrm{P}>0.05$ vs. parental cells. RhoA-KD, RhoA-knockdown.

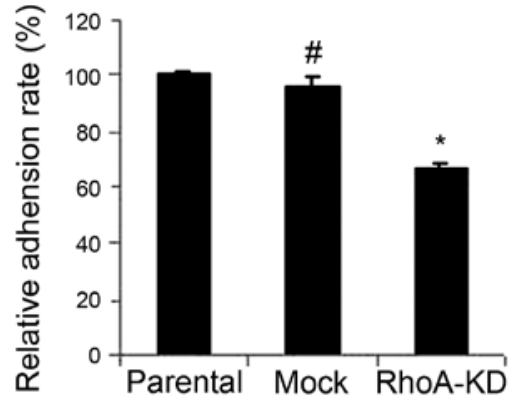

Figure 3. Impact of RhoA knockdown on tumor cell adhesion ability. Stable RhoA-KD, mock and parental HO8910 cells were grown and subjected to the tumor cell adhesion assay. The relative adhesion rate was significantly lower in the RhoA-KD cells than in the parental and mock cells $(\mathrm{P}<0.05)$. Data are presented as the mean $\pm \mathrm{SD}$ of three independent experiments. ${ }^{*} \mathrm{P}<0.05$ vs. parental and mock cells; ${ }^{\prime} \mathrm{P}>0.05$ vs. parental cells. RhoA-KD, RhoA-knockdown.

sluggish, but their abdominal circumference was obviously increased. By contrast, the nude mice with the RhoA-KD cell injection were generally in good condition and their abdominal circumference was gradually increased compared to the remaining two groups (Fig. $4 \mathrm{~A}, \mathrm{P}<0.05$ ). Moreover, when the mice were sacrificed at 4 weeks post-inoculation, the tumor formation rate was $71.4 \%(5 / 7)$ in the RhoA-KD group compared to $100 \%(7 / 7)$ in the remaining two groups. The volume of ascites, number of tumor lesions, total number of disseminated tumor nodules, and tumor weight were all significantly smaller in the RhoA-KD group than in the remaining two groups (Fig. 4B, $\mathrm{P}<0.05$ ). However, there was no statistically significant difference in tumor growth between the parental and mock groups $(\mathrm{P}>0.05)$. In addition, as shown in Fig. 5A, there were more necrotic and apoptotic regions found in tumors with RhoA knockdown than in the parental and mock groups. We performed a TUNEL assay to detect the apoptotic level in these tumor xenografts and found that the apoptotic index was significantly higher in the RhoA-KD group than in the parental and mock groups (Fig. 5E, $\mathrm{P}<0.05$ ). However, there was no statistically significant difference observed between the parental and mock groups (Fig. 5E, P>0.05).

We confirmed RhoA knockdown in the mouse tumor xenografts using RT-qPCR, western blot analysis and immunostaining of RhoA mRNA and protein, respectively. The results showed that the RhoA mRNA level was significantly reduced in the RhoA-KD group, compared to the parental and mock groups (Fig. 5B, $\mathrm{P}<0.05)$. Similarly, the RhoA protein level was significantly reduced in the RhoA-KD group compared with the parental and mock groups (Fig. 5C and $\mathrm{D}, \mathrm{P}<0.05$ ). However, there was no significant difference of RhoA expression between the parental and mock groups $(\mathrm{P}>0.05)$. 
A

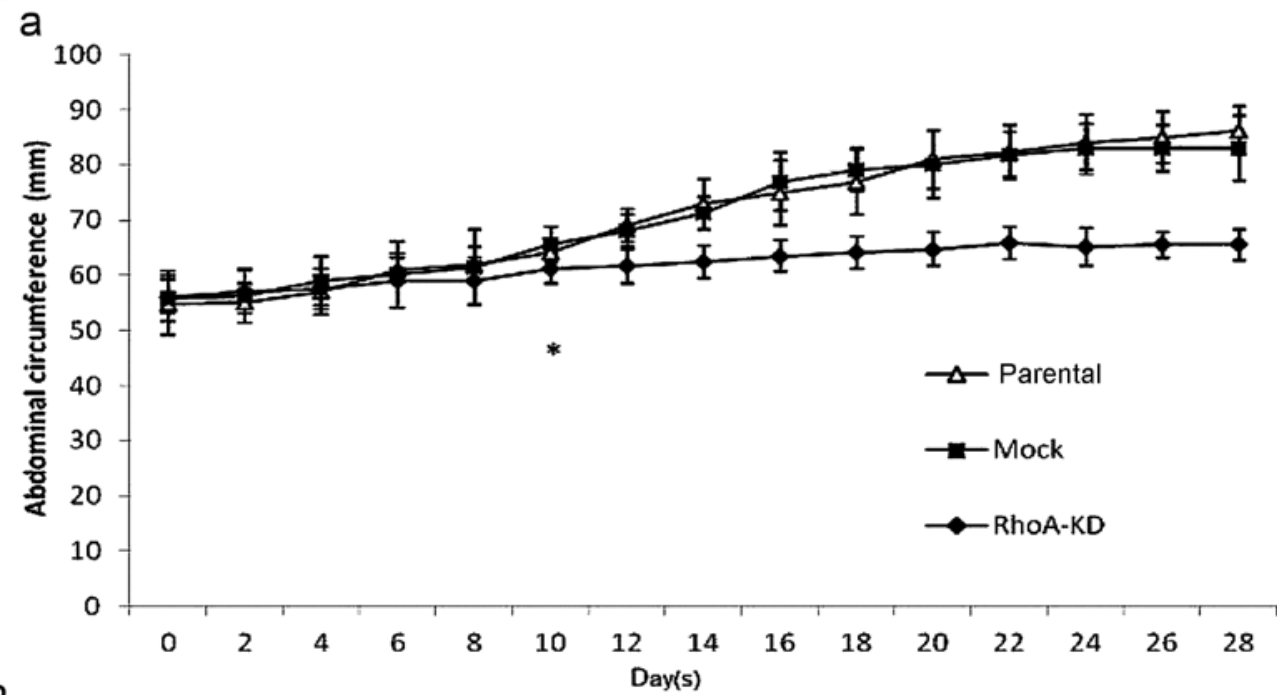

$\mathrm{B}$

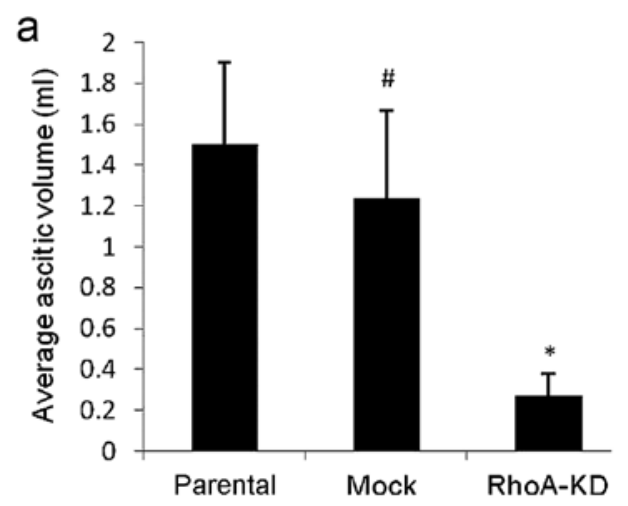

b

C

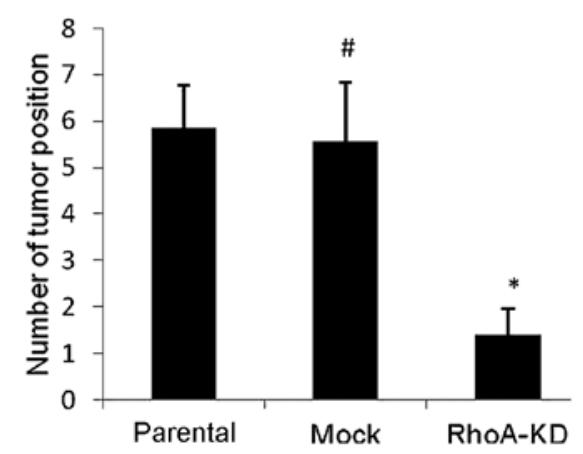

d
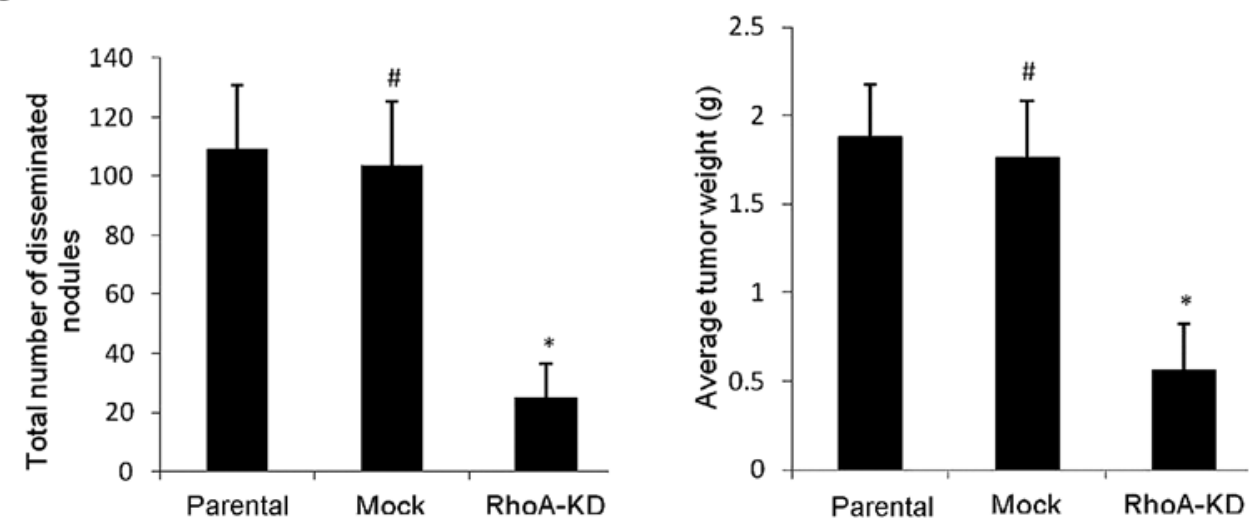

Figure 4. Effects of RhoA knockdown on the regulation of tumor xenograft formation and growth in a nude mouse intraperitoneal tumorigenicity model (A) Measurement of abdominal circumferences of nude mice. (a) Abdominal circumference growth curve was plotted against time elapsed (P<0.05, from this point onwards). The results show that the abdominal circumference from the RhoA-KD-bearing mice was much smaller than that of the parental and mock groups $(\mathrm{P}<0.05)$, whereas there was no significantly difference between the mock and parental groups $(\mathrm{P}>0.05)$. (B) Measurement of tumor burdens. Tumor cells were disseminated in the abdominal cavities of the three groups of nude mice (data not shown). Numerous tumor nodules were disseminated on the peritoneal surface, stomach, diaphragm, colic omentum, mesentery, liver, spleen or kidney of the parental and mock groups, whereas few tumor nodules were shown on the peritoneal surface and around the radix of mesentery in the RhoA-KD group. (a) Volume of ascites. (b) Number of tumor-disseminated position. (c) Total number of tumor nodules. (d) Tumor weight. These parameters were shown to be significantly reduced in the RhoA-KD group compared to the parental and mock groups $(\mathrm{P}<0.05)$. Data are presented as means $\pm \mathrm{SD} .{ }^{*} \mathrm{P}<0.05$ vs. parental and mock groups; ${ }^{\#} \mathrm{P}>0.05$ vs. parental cells. RhoA-KD, RhoA-knockdown.

\section{Discussion}

Epithelial ovarian cancer is frequently diagnosed at the advanced stages of disease. Thus, it is a lethal gynecological malignancy and only 5-30\% of ovarian cancer patients survive for 5 years $(6,24)$. Tumor peritoneal metastasis occurs most frequently $(6,24)$. In the present study, we targeted RhoA in ovarian cancer cells to further assess the role of RhoA knockdown in the regulation of ovarian cancer cell biological behaviors in vitro and in a nude mouse model. We found that 
A
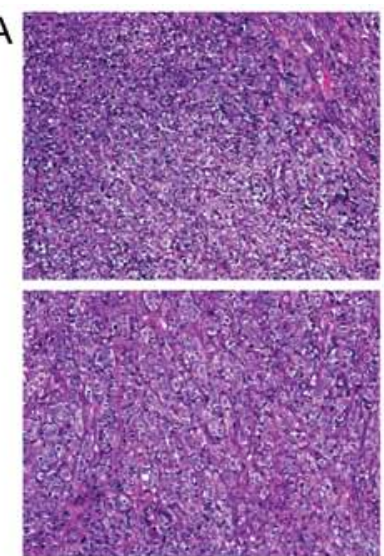

Parental

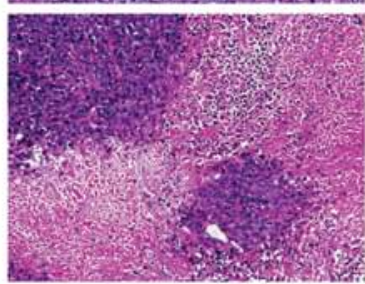

Mock

C



RhoA-KD

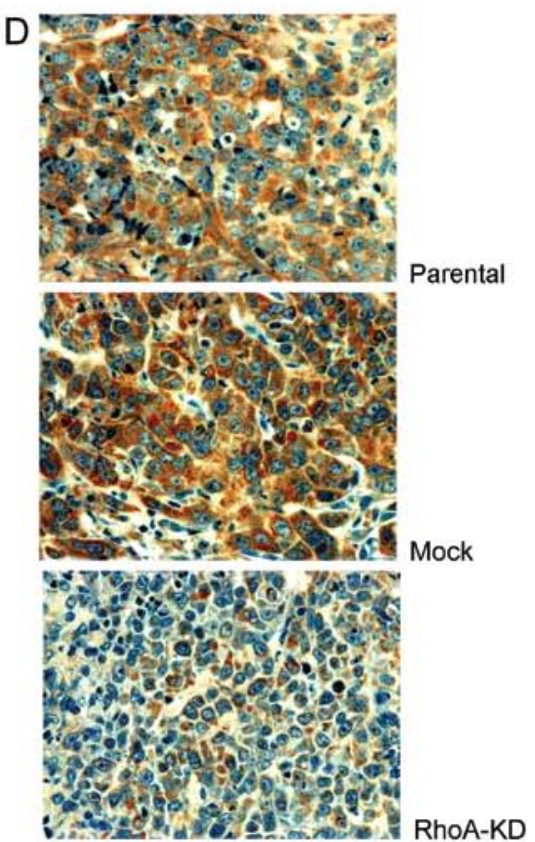

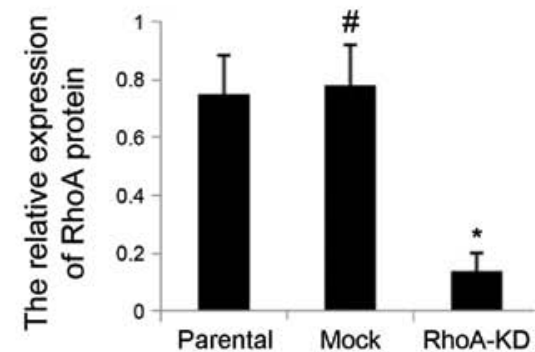

B

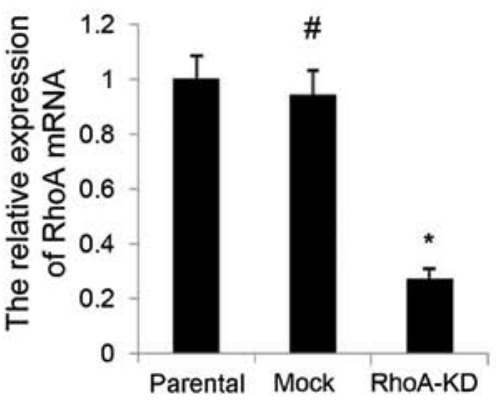

$\mathrm{E}$

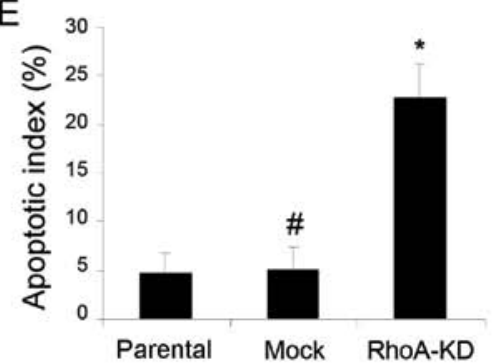

Figure 5. Effects of RhoA knockdown on the regulation of tumor cell biological behaviors in nude mice. (A) Hematoxylin and eosin staining of tumor xenografts. (B) RT-qPCR. Tumor xenografts were removed from the nude mice on day 28 after tumor cell injection and subjected to RNA isolation and qRT-PCR analysis of the RhoA mRNA level. The results show a marked decrease in the RhoA mRNA level in the RhoA-KD group xenografts $(\mathrm{P}<0.05)$. (C) Western blot analysis. Tumor xenografts were then subjected to western blot analysis of RhoA protein. The graph shows the quantified data of the western blot analysis results, revealing a significant reduction of RhoA protein in the RhoA-KD group xenografts $(\mathrm{P}<0.05)$. (D) Immunohistochemistry. Tumor xenografts were subjected to tissue processing and immunostaining of RhoA protein. The results also show that the tumor cells were stained very weakly with the RhoA antibody in the RhoA-KD group compared to those of the parental and mock groups (magnification, $\mathrm{x} 400$ ). Data are presented as the mean $\pm \mathrm{SD}$. "P<0.05 vs. parental and mock cells; ${ }^{~} \mathrm{P}>0.05$ vs. parental cells. (E) TUNEL assay. Tumor xenografts were subjected to the TUNEL assay to detect tumor cell apoptosis. The graph shows the apoptotic index, which was significantly increased in the tumor sections of the RhoA-KD group compared to those of the parental and mock groups $(\mathrm{P}<0.05)$. The data are presented as the average apoptotic index $\pm \mathrm{SD}$. ${ }^{*} \mathrm{P}<0.05$ vs. parental and mock cells; ${ }^{*} \mathrm{P}>0.05$ vs. parental cells. RhoA-KD, RhoA-knockdown; RT-qPCR, reverse transcription-quantitative polymerase chain reaction; TUNEL, terminal deoxynucleotidyltransferase-mediated dUTP nick end-labeling. 
RhoA knockdown significantly reduced tumor cell viability, migration, invasion, and adhesion abilities in vitro and inhibited tumor cell settling in the abdominal cavity and tumor formation in nude mice. The residual tumor xenografts showed necrosis and apoptosis after RhoA knockdown. The results of the present study confirmed RhoA activity in ovarian cancer. Future studies may target RhoA activity as a novel strategy to treat ovarian cancer patients.

RhoA is a member of the Ras gene superfamily of small GTPases. The biological function of RhoA is controlled by the cycling between the active GTP-bound and inactive GDP-bound states (25). Early evidence has shown that RhoA is able to modulate cell adhesion, contraction, mobility and degradation of the extracellular matrix. However, more recent studies have clearly demonstrated that RhoA affects cell proliferation, angiogenesis, gene expression and tumor cell invasion and metastasis $(26,27)$. For example, Rho proteins must be present for cells to progress through the G1 phase of the cell cycle (28). In experimental models of carcinogenesis, aberrant RhoA activation induced cell growth, transformation, invasion and metastasis (29-32). Molecularly, RhoA regulates the activities of multiple transcription factors, such as ROCK, Cdc 42 and Rac1, most of which are involved in cancer (10). The upregulation of RhoA expression or activity has been associated with tumor progression (15), whereas the downregulation of RhoA expression or activity has been shown to promote apoptosis of gastric cancer cells and inhibit the growth and invasion of gastric cancer cells in vitro (19). Furthermore, factors such as epidermal growth factor and lysophosphatidic acid, are able to activate RhoA protein $(29,32)$, further indicating the role of RhoA in tumorigenesis.

A previous study has shown that RhoA expression was significantly higher in metastatic omentum than in ovarian cancer, benign tumors, and normal fallopian tube epithelium and is associated with poor tumor differentiation and advanced stages of ovarian cancer (33). In addition, an in vitro study has demonstrated that inhibition of the Rho/ROCK pathway enhanced the efficacy of cisplatin in human ovarian cancer cells (34). By contrast, Rho expression was higher in patients who did not respond to chemotherapy (35). In the present study, the results on RhoA knockdown in ovarian cancer cells in vitro supported the previous findings $(15,19)$. Our results using the nude mouse intraperitoneal tumorigenicity model specifically showed that RhoA knockdown was able to suppress tumor formation and growth in the abdominal cavity, which is the site where ovarian cancer frequently spreads. A recent review indicated that RhoA is a therapeutic target for ovarian cancer (36), while another study showed that the overexpression of RhoA enhanced peritoneal dissemination and that RhoA suppression with lovastatin may be a useful therapy for ovarian cancer (37).

In the present study, shRNA, made of a tight hairpin turn of RNA sequences, was used to silence the target gene expression via RNA interference, which is a well-established technique to inhibit gene expression, typically by causing the destruction of specific mRNA molecules with high efficiency and specificity as well as low toxicity (38). In recent years, lentivirus-mediated shRNA has been used successfully in vitro and in vivo and has shown great promise in the field of cancer therapy (39). Thus, in the present study, we used this technique to knock down RhoA expression in ovarian cancer HO8910 cells, a highly invasive human ovarian cancer cell line with a high level of RhoA expression (17). The results have shown that this lentivirus may silence RhoA expression with high efficiency. However, our results are preliminary and a proof-of-principle study as the side effects of this lentivirus in vivo were not assessed. In addition, we did not examine any molecular events after RhoA knockdown. Thus, future studies should focus on the underlying mechanisms of the antitumor effects in RhoA-knockdown tumor cells.

\section{Acknowledgements}

This study was supported in part by a grant from the Scientific and Technological program of Guangdong Province, China (no. 2010B031600185). This manuscript has been edited and proofread by Medjaden Bioscience Limited.

\section{References}

1. Lozano R, Naghavi M, Foreman K, Lim S, Shibuya K, Aboyans V, Abraham J, Adair T, Aggarwal R, Ahn SY, et al: Global and regional mortality from 235 causes of death for 20 age groups in 1990 and 2010: a systematic analysis for the Global Burden of Disease Study 2010. Lancet 380: 2095-2128, 2012.

2. Moyer VA; USA Preventive Services Task Force: Screening for ovarian cancer: USA Preventive Services Task Force reaffirmation recommendation statement. Ann Intern Med 157: 900-904, 2012.

3. Vo C and Carney ME: Ovarian cancer hormonal and environmental risk effect. Obstet Gynecol Clin North Am 34: 687-700, 2007.

4. Hunn J and Rodriguez GC: Ovarian cancer: etiology, risk factors, and epidemiology. Clin Obstet Gynecol 55: 3-23, 2012.

5. Gilbert L, Basso O, Sampalis J, Karp I, Martins C, Feng J, Piedimonte S, Quintal L, Ramanakumar AV, Takefman J, et al; DOvE Study Group: Assessment of symptomatic women for early diagnosis of ovarian cancer: results from the prospective DOvE pilot project. Lancet Oncol 13: 285-291, 2012.

6. Hennessy BT, Coleman RL and Markman M: Ovarian cancer. Lancet 374: 1371-1382, 2009.

7. Hu J, Shao S, Song Y, Zhao J, Dong Y, Gong L and Yang P: Hepatocyte growth factor induces invasion and migration of ovarian cancer cells by decreasing the expression of E-cadherin, beta-catenin, and caveolin-1. Anat Rec (Hoboken) 293: 1134-1139, 2010.

8. Fader AN and Rose PG: Role of surgery in ovarian carcinoma. J Clin Oncol 25: 2873-2883, 2007.

9. Gur S, Kadowitz PJ and Hellstrom WJ: RhoA/Rho-kinase as a therapeutic target for the male urogenital tract. J Sex Med 8: 675-687, 2011.

10. Rathinam R, Berrier A and Alahari SK: Role of Rho GTPases and their regulators in cancer progression. Front Biosci (Landmark Ed) 16: 2561-2571, 2011

11. Kwiatkowska A and Symons M: Signaling determinants of glioma cell invasion. Adv Exp Med Biol 986: 121-141, 2013.

12. Oh HK, Sin JI, Choi J, Park SH, Lee TS and Choi YS: Overexpression of CD73 in epithelial ovarian carcinoma is associated with better prognosis, lower stage, better differentiation and lower regulatory $\mathrm{T}$ cell infiltration. J Gynecol Oncol 23: 274-281, 2012.

13. Takami Y, Higashi M, Kumagai S, Kuo PC, Kawana H, Koda K, Miyazaki $M$ and Harigaya K: The activity of RhoA is correlated with lymph node metastasis in human colorectal cancer. Dig Dis Sci 53: 467-473, 2008.

14. Zhao X, Lu L, Pokhriyal N, Ma H, Duan L, Lin S, Jafari N, Band $\mathrm{H}$ and Band V: Overexpression of RhoA induces preneoplastic transformation of primary mammary epithelial cells. Cancer Res 69: 483-491, 2009.

15. Friesland A, Zhao Y, Chen YH, Wang L, Zhou H and Lu Q: Small molecule targeting Cdc42-intersectin interaction disrupts Golgi organization and suppresses cell motility. Proc Natl Acad Sci USA 110: 1261-1266, 2013. 
16. Li J, Tang LD, Tang WX and Ng SW: Expression and significance of RhoA protein in epithelial ovarian cancer cells. J Endocr Surg 3: 147-153, 2008. (In Chinese).

17. Xing L, Yao X, Williams KR and Bassell GJ: Negative regulation of RhoA translation and signaling by hnRNP-Q1 affects cellular morphogenesis. Mol Biol Cell 23: 1500-1509, 2012.

18. Shimada T, Nishimura Y, Nishiuma T, Rikitake Y, Hirase T and Yokoyama M: Adenoviral transfer of rho family proteins to lung cancer cells ameliorates cell proliferation and motility and increases apoptotic change. Kobe J Med Sci 53: 125-134, 2007

19. Wang H, Zhao G, Liu X, Sui A, Yang K, Yao R, Wang Z and Shi Q: Silencing of RhoA and RhoC expression by RNA interference suppresses human colorectal carcinoma growth in vivo. J Exp Clin Cancer Res 29: 123, 2010.

20. Li QQ, Skinner J and Bennett JE: Evaluation of reference genes for real-time quantitative PCR studies in Candida glabrata following azole treatment. BMC Mol Biol 13: 22, 2012.

21. Ali HM, Urbinati G, Raouane M and Massaad-Massade L: Significance and applications of nanoparticles in siRNA delivery for cancer therapy. Expert Rev Clin Pharmacol 5: 403-412, 2012

22. Kelly P, Stemmle LN, Madden JF, Fields TA, Daaka Y and Casey PJ: A role for the G12 family of heterotrimeric G proteins in prostate cancer invasion. J Biol Chem 281: 26483-26490, 2006.

23. Kelly P, Moeller BJ, Juneja J, Booden MA, Der CJ, Daaka Y, Dewhirst MW, Fields TA and Casey PJ: The G12 family of heterotrimeric $\mathrm{G}$ proteins promotes breast cancer invasion and metastasis. Proc Natl Acad Sci USA 103: 8173-8178, 2006.

24. Basu Roy UK, Henkhaus RS, Loupakis F, Cremolini C, Gerner EW and Ignatenko NA: Caveolin-1 is a novel regulator of K-RAS-dependent migration in colon carcinogenesis. Int $\mathrm{J}$ Cancer 133: 43-57, 2013.

25. Nikonova E, Tsyganov MA, Kolch W, Fey D and Kholodenko BN: Control of the G-protein cascade dynamics by GDP dissociation inhibitors. Mol Biosyst 9: 2454-2462, 2013.

26. Struckhoff AP, Rana MK and Worthylake RA: RhoA can lead the way in tumor cell invasion and metastasis. Front Biosci (Landmark Ed) 16: 1915-1926, 2011.

27. Fiordalisi JJ, Keller PJ and Cox AD: PRL tyrosine phosphatases regulate rho family GTPases to promote invasion and motility. Cancer Res 66: 3153-3161, 2006
28. Olson MF, Ashworth A and Hall A: An essential role for Rho, Rac, and dc42 GTPases in cell cycle progression through G1. Science 269: 1270-1272, 1995.

29. Burridge $\mathrm{K}$ and Wennerberg $\mathrm{K}$ : Rho and Rac take center stage. Cell 116: 167-179, 2004

30. Gómez del Pulgar T, Benitah SA, Valerón PF, Espina C and Lacal JC: Rho GTPase expression in tumourigenesis: evidence for a significant link. BioEssays 27: 602-613, 2005.

31. Qiu RG, Chen J, McCormick F and Symons M: A role for Rho in Ras transformation. Proc Natl Acad Sci USA 92: 11781-11785, 1995.

32. Sahai E and Marshall CJ: RHO-GTPases and cancer. Nat Rev Cancer 2: 133-142, 2002.

33. Chen S, Wang J, Gou WF, Xiu YL, Zheng HC, Zong ZH, Takano $Y$ and Zhao Y: The involvement of RhoA and Wnt-5a in the tumorigenesis and progression of ovarian epithelial carcinoma. Int J Mol Sci s14: 24187-24199, 2013.

34. Ohta T, Takahashi T, Shibuya T, Amita M,Henmi N, Takahashi K and KurachiH: Inhibition of the Rho/ROCK pathway enhances the efficacy of cisplatin through the blockage of hypoxia-inducible factor- $1 \alpha$ in human ovarian cancer cells. Cancer Biol Ther 13: 25-33, 2012.

35. Canet B, Pons C, Espinosa I and Prat J: Ovarian clear cell carcinomas: RHO GTPases may contribute to explain their singular biologic behavior. Hum Pathol 42: 833-839, 2011.

36. Gest C, Mirshahi P, Li H, Pritchard LL, Joimel U, Blot E, Chidiac J, Poletto B, Vannier JP, Varin R, et al: Ovarian cancer: Stat3, RhoA and IGF-IR as therapeutic targets. Cancer Lett 317: 207-217, 2012.

37. Horiuchi A, Kikuchi N, Osada R, Wang C, Hayashi A, Nikaido T and Konishi I: Overexpression of RhoA enhances peritoneal dissemination: RhoA suppression with lovastatin may be useful for ovarian cancer. Cancer Sci 99: 2532-2539, 2008.

38. Boudreau RL and Davidson BL: Generation of hairpin-based RNAi vectors for biological and therapeutic application. Methods Enzymol 507: 275-296, 2012

39. Khatri N, Rathi M, Baradia D, Trehan S and Misra A: In vivo delivery aspects of miRNA, shRNA and siRNA. Crit Rev Ther Drug Carrier Syst 29: 487-527, 2012. 